\title{
EFFECTS OF DYNAMIC ForCES AND STRAIN RATE DURING BATTERY-ELECTRIC BUS IMPACT
}

\author{
Stanislav Spirk, Miloslav Kepka \\ University of West Bohemia, Faculty of Mechanical Engineering, Regional Technological Institute \\ Univerzitni 8, 30614 Pilsen, Czech Republic
}

\begin{abstract}
Electromobility is a rapidly growing area of transport. Construction of new electric vehicles is associated with new approaches and with finding new solutions. The REESS (Rechargeable Energy Storage System) is a very heavy component installed in battery-electric buses. Its safety during mechanical impact must be verified experimentally. Therefore, it is practical to use computer simulation as early as the vehicle design stage to obtain a preliminary estimate of the REESS behaviour when subjected to a mechanical shock. Using computer simulations, various factors can be explored. The present paper describes examples of simulations used in the development of a new battery-electric bus. Effects of dynamic (impact) loads and behaviour of materials at higher strain rates are studied.
\end{abstract}

Keywords battery-electric bus; rechargeable energy storage system; computer simulation; FEM; dynamic impact loading; strain rate;
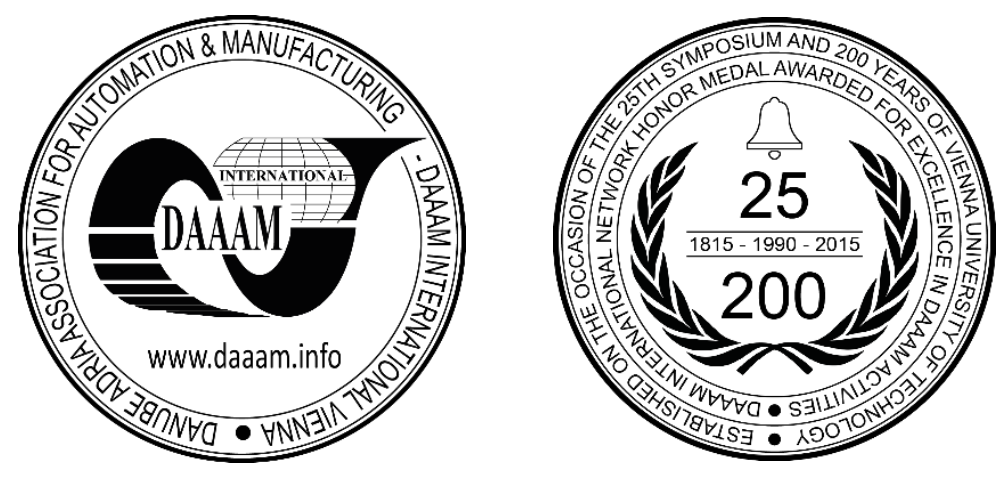

This Publication has to be referred as: Spirk, S[tanislav] \& Kepka, M[iloslav] (2016). Effects of Dynamic Forces and Strain Rate During Battery-Electric Bus Impact, Proceedings of the 26th DAAAM International Symposium, pp.09200925, B. Katalinic (Ed.), Published by DAAAM International, ISBN 978-3-902734-07-5, ISSN 1726-9679, Vienna, Austria

DOI: $10.2507 / 26$ th.daaam.proceedings. 128 


\section{Introduction}

The present paper discusses the effects that occur during an electric bus crash. Current trends in mass transport clearly favour zero-emission technologies. The European Union is undertaking an intensive effort to support low-carbon strategies, most notably in urban areas. According to plans, one half of municipal mass transport vehicles will be propelled by technologies other than the combustion engine by 2030. The electric operated bus today and in the future is classified as an environmentally-friendly and energy saving transportation system; locally emission free thanks to the electric drive, low-noise, and gentle but also powerful during start and stop. [10] In electric buses, certain problems arise which are not encountered in conventional vehicles. The passive safety of electric buses is a specific issue due to the presence of the REESS. In vehicle side impact, thanks to the cushioning effect of the battery tank door and the bus frame, the battery system is not hit directly, which reduces the harm to the battery and protects the battery from squashing and danger. [3] The behaviour of batteries alone upon a collision with another object (a barrier, part of another vehicle or other objects) can be studied as well. The purpose of such studies is to obtain a validated model of batteries usable in further calculations. Developing reliable finite-element deformation and failure models of the battery for the vehicle design process is a must. [4] Another problem relates to the strength of attachment of the batteries (and the REESS). Batteries are rather heavy units with large inertial forces occurring upon a collision of the electric bus. The points of attachment of the REESS (which contains such heavy batteries) pose a significant safety risk. For this purpose, simulations of the REESS involving the surrounding structure have been developed. The models for these simulations were based on CAD data from a local developer. The simulations were complemented with experimental verification of material properties.

\section{Problem description}

\subsection{Requirements}

In this context, the fundamental document is the Regulation No. 100 (Revision 2) "Uniform provisions concerning the approval of vehicles with regard to specific requirements for the electric power train" [9]. This regulation sets out the requirements for installed electrical equipment and, above all, the safety requirements for the REESS. It lists a number of technical parameters and describes methods of their testing. The regulation also defines the requirements for measuring the isolation resistance of REESS components. It deals with vibration tests which map the safety of the REESS in a vibrating environment. It also covers the safety aspects of thermal shock and cycling and prescribes a fire resistance test.

\subsection{Problem specification}

The main purpose of this investigation relates to the strength of the REESS upon the collision of an electric bus with a heavy barrier. Because experimental testing of the crashworthiness of the REESS is costly, its behaviour in a crash is studied using computer simulations. The present solution uses design data for S̆KODA Perun HE electric bus, a vehicle which is already used in service [1]. It is an M3-category vehicle. The capacity of its batteries (Li-Pol Nickel Manganese Cobalt Oxide) is $221 \mathrm{kWh}$ and its range is up to $150 \mathrm{~km}$. With a 160-kW electric motor, the electric bus can reach speeds of $70 \mathrm{kph}$. The electrical energy needed is stored in three REES systems. One of them is in the front part of the electric bus (behind the driver cab) and the other two are located in the rear (Fig. 1). Each REESS comprises three slide-in modules with six battery units. The mass of a single REESS is $750 \mathrm{~kg}$. Each REESS is attached to the vehicle structure with six M16 screws located in its bottom part and two M10 screws in its top part. At the bottom, the electric bus structure at the point of attachment of the REESS is reinforced with a metal sheet of $5 \mathrm{~mm}$ thickness. At the top, the REESS and the electric bus structure are attached with brackets.

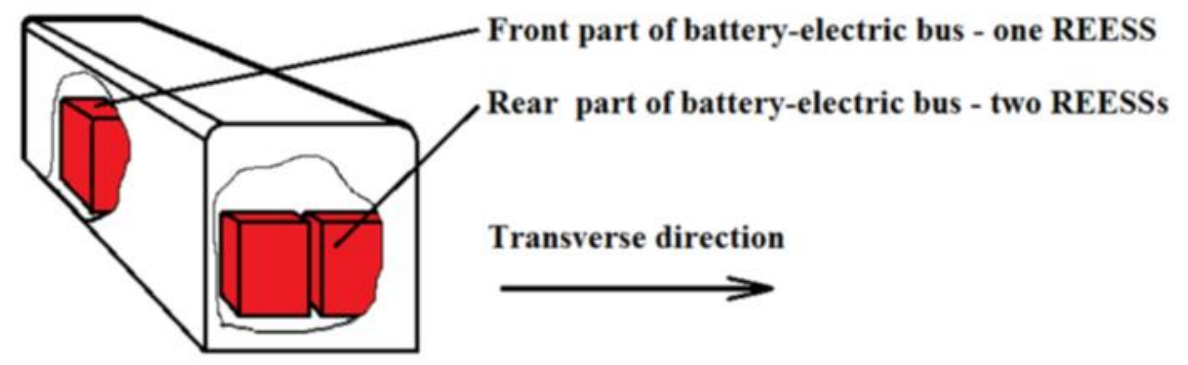

Fig. 1. REESS locations in the electric bus

All parts of the electric bus are subject to gravitation and, upon impact, the impact acceleration pulse. The acceleration pulse that acts on the REESS is clearly defined in the UN/ECE 100.2 regulation [9]. For electric buses (the M3 vehicle category), the acceleration pulse parameters are prescribed. The minimum acceleration is $5 \mathrm{~g}$ and the maximum 
acceleration is $12 \mathrm{~g}$. The test acceleration must be within this interval. For the present simulations the maximum acceleration levels have been used.

\subsection{FEM methodology}

The start of the development of explicit solvers dates back to 1960s. Solvers that rely on explicit and implicit FEM use different methods of time integration for solving dynamic problems. The explicit method is a clearly more suitable choice for vehicle collisions because the explicit time integration is appropriate for simulating processes that involve large strains and shape changes. Generally, explicit solvers are better suited for problems with complex contact conditions. In addition, the computation is much faster, as the inversion of the stiffness matrix is bypassed. In this case, any nonlinearity is included in the values of nodal forces.

The explicit code is essentially derived from Newton's second law of motion. It is a motion equation in a matrix form. This equation is defined at a particular time instant. For the equilibrium of dynamic forces to be reached, the expression below must be valid. [5]

$$
M \dot{v}=f^{e x t}-f^{i n t}
$$

Here, $\mathrm{M}$ is the mass matrix; $\mathrm{f}^{\text {int }}$ and $\mathrm{f}^{\text {ext }}$ are nodal force matrices given by the external and internal resistance of the element, and $\dot{v}$ stands for acceleration. This relationship can be expressed in matrix form and defined at a particular time instant.

$$
\left\{F_{t}^{\text {int }}\right\}=\sum\left(\int_{\Omega}[B]^{T}\left\{\sigma_{n}\right\} d \Omega+\left\{F^{\text {houg }}\right\}\right)+\left\{F^{\text {cnt }}\right\}
$$

Further, $\left\{\sigma_{n}\right\}$ is the internal stress matrix and [B] is the matrix of shape change elements. The $\left\{F^{\text {houg }}\right\}$ term has been added to prevent the hourglassing effect and the $\left\{F^{c n t}\right\}$ is the contact force vector. A great advantage of the explicit method is the use of elements with a single integration point. They are well suited for large strains and their use shortens the computation time. The energy and stress of the element are only evaluated for a single integration point. However, the advantages of this method come at the cost of reduced computation stability. The result of the computation is then marked by the typical imbalance between the kinetic and the internal energy of the system. This numeric error is known as hourglassing. During calculation, the total energy must be checked continuously. The critical limit is believed to be the increase in the hourglass energy above $5 \%$ of the total energy of the system.

Explicit code-based solvers are conditionally stable. The main aspect is the magnitude of the time step $t_{\text {calc }}$. It is related to the propagation of stress waves through the material. The critical time step $t_{\text {crit }}$ can be determined from the time interval, in which the front of the stress wave passes through the element.

$$
t_{\text {calc }} \leq t_{\text {crit }}=\frac{l}{c}=l \sqrt{\frac{\rho}{E}}
$$

Here, $\mathrm{c}$ is the velocity of propagation of stress waves through the material, 1 is the characteristic dimension of the element, $\mathrm{E}$ is the modulus of elasticity of the material and $\rho$ denotes the material density.

The tool used in the present study was the Pam-Crash solver. This FEM solver is part of the VPS (Virtual Performance Solution) software package from ESI Group. [1] The software is used for simulating impact and for assessing the safety of passengers. It is most widely used in the automotive industry. Its development dates back to 1978 and is related to the first simulations of crash tests of automobiles. Based on the finite element method (FEM), it allows complex geometries to be modelled, thanks to many available types of elements. It offers a broad range of both linear and nonlinear materials, including elastic and visco-plastic materials, foams, multi-layer composites and failure and defect models. [2] With its use of explicit FEM, it is suitable for non-linear problems with large numbers of contacts. Many types of contacts are available, particularly with the penalty method.

\subsection{Physical model description}

The FEM model is not intended to provide a description of the behaviour of the entire bus. The units used here include the following: $\mathrm{mm}, \mathrm{kg}, \mathrm{ms}$, (kN, GPa). The focus is on the front REESS crashworthiness and its attachment during a collision. It is clear that in order to assess the behaviour of joints, parts of the electric bus structure adjacent to them must be included in the model. It is impossible to obtain a relevant description of the structure's behaviour in the vicinity of the joint using an ideal attachment of the REES system to a rigid body. When only a portion of the electric bus body is studied, the problem can be divided into two parts. Then, the behaviour of the REESS in the front part of the vehicle (Fig. 2) can be explored separately.

The fundamental component in both models (front and rear part of the electric bus) is the model of the REESS. It was designed for exploring both longitudinal and transverse accelerations. Each REESS contains three wheeled slidein modules made of Ertalon 6SA material. The front part of the slide-in module is held in place with screws, while the 
rear part presses against stops. The wheels are included in the model as supplementary non-rotating load-carrying components but their friction coefficient value corresponds to rolling motion. Each battery pack is an independent rigid body with defined mass $(30 \mathrm{~kg})$ and moments of inertia. The batteries are held in place inside the slide-in module by strips of Lamplex material. The model of the entire battery box does not include the sheet metal covers. These covers are attached to the box with rivets of limited load-carrying capacity. All steel parts of the battery box (including the slide-in modules) are made of S235 steel.

After preliminary assessment of the computing time, the initial element size was set at $10 \mathrm{~mm}$ for all meshes. In all shell elements, five integration points across the thickness have been used. Welds are included in the model as mesh point connections. Symmetric node-to-segment contacts have been employed. The contact thickness was defined so that the clearance between the parts in contact matched the real-world conditions. There is no initial penetration in the model. A single REESS contains 48 of these contact types. The screws are ideally represented as $1 \mathrm{D}$ elements where subtraction of acting forces is enabled. All degrees of freedom have been removed from the outermost nodes of the structure. The structure is subject to the acceleration pulse (paragraph 2.1 and 2.2) and to gravitational acceleration.

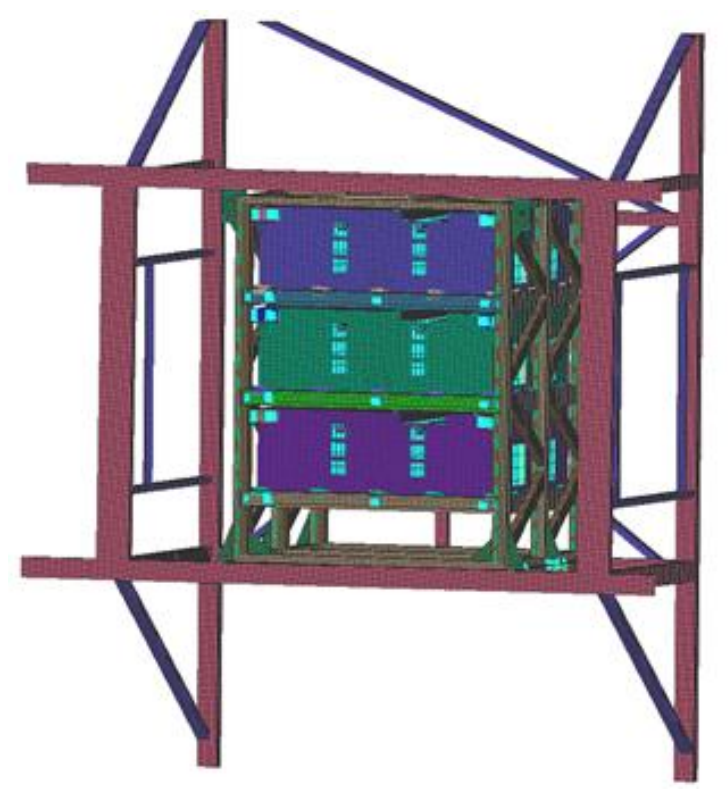

Fig. 2. Model of the REESS - the front part of the electric bus;

To improve the accuracy of computation, it is desirable to measure the mechanical properties of the materials. Tests of the three steels listed below have been conducted. The structure of the REESS is made of the S235 material [6]. The structure of the electric bus is made of the 1.4301 material [7], with brackets made of S355 [8]. As the structures of the electric bus and of the battery boxes comprise many welded joints, mechanical properties of the materials altered by welding should be mapped as well. For this purpose, welded specimens of the three steels have been made. The parameters for the welding processes were provided by manufacturers of all the relevant components. When such test specimens are welded, the process should be as close as possible to the real-world procedure. The welding procedures used for the materials are outlined below.

- S235, sheet thickness: 3 mm (REESS structure)

Welded according to EN 287 - 1135 P BW 1.1 B t10.0 PF ssnb

Welding wire OK AristoRod 12.50 1dia. EN ISO - 14341 - AG3833C1 Manufacturer: ESAB

- $\quad \mathbf{1 . 4 3 0 1}$ sheet thickness 3 mm (electric bus body)

135 MAG material ER $3071 \mathrm{~mm}$

- $\quad$ S355, sheet thickness: 5 mm (brackets for REESS attachment)

MAG - in ARGON + CO2 (92\%-18\%) shielding gas or CORGON 18 gas supplied by LINDE, filler rod 1.2 (dia.) G3Si1

Results of tests (stress-strain curves) for steels without welds (Fig. 3) contain true stress and true strain data. With each material, tests were conducted at four loading rates (strain rate $=0.015,1.5,15,150 \mathrm{~s}-1$ ). It is thus possible to account for work hardening during rapid deformation. Mechanical properties were measured using tension and microtension tests. Deformation of the test pieces was recorded using a contact extensometer. 


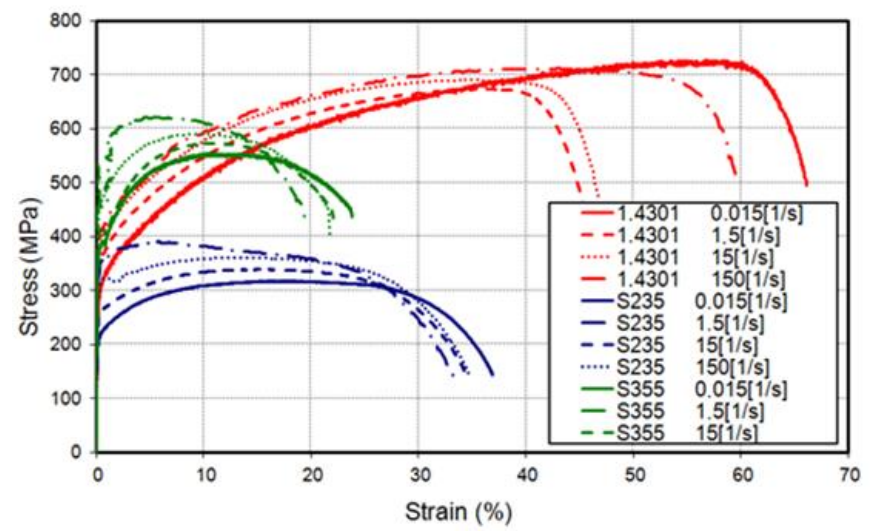

Fig. 3. Tension test plots for materials without welds

The welded specimens were tested in a similar manner using the strain rates of $0.01,0.1,1 \mathrm{~s}-1$. The change in mechanical properties upon welding is reflected in the difference between the stress-strain curves for materials before and after welding. Upon welding, all materials showed slight embrittlement. The material that was affected most severely by welding was the 1.4301 steel. Its failure occurred at a lower strain. It is therefore advisable to define smaller permissible deformation in the weld region for evaluating the simulations.

\section{Effect of strain rate}

Higher strain rates during loading induce work-hardening in steel materials. The strain rate behaviour is well described thanks to the above experiments. The present material models are based on those data. The material behaviour was explored for strain rates in the defined range $\left(0.015,1.5,15,150 \mathrm{~s}^{-1}\right)$. The strain rates used in the simulations were approximately $50 \mathrm{~s}^{-1}$. Two material models were constructed for exploring the effects of strain rate. The first model described the material without taking into account its strain rate behaviour. The second was based on the newly-defined material models. The resultant plastic von Mises stress was $0.522 \mathrm{GPa}$ with the model which lacked the strain rate response. In models with the strain rate behaviour accounted for, von Mises stress was $0.501 \mathrm{GPa}$. This difference is quite small (see Fig. 4) and its visibility in the figures is affected by the chosen ranges of the stress scale. It is less notable when the same ranges are used. The differences between maximum plastic strain levels through thickness are much easier to see. The maximum plastic strain is $0.195 \mathrm{~mm} / \mathrm{mm}$ for the model without the strain rate response. In models with the strain rate behaviour included, the max. plastic strain was $0.155 \mathrm{~mm} / \mathrm{mm}$.
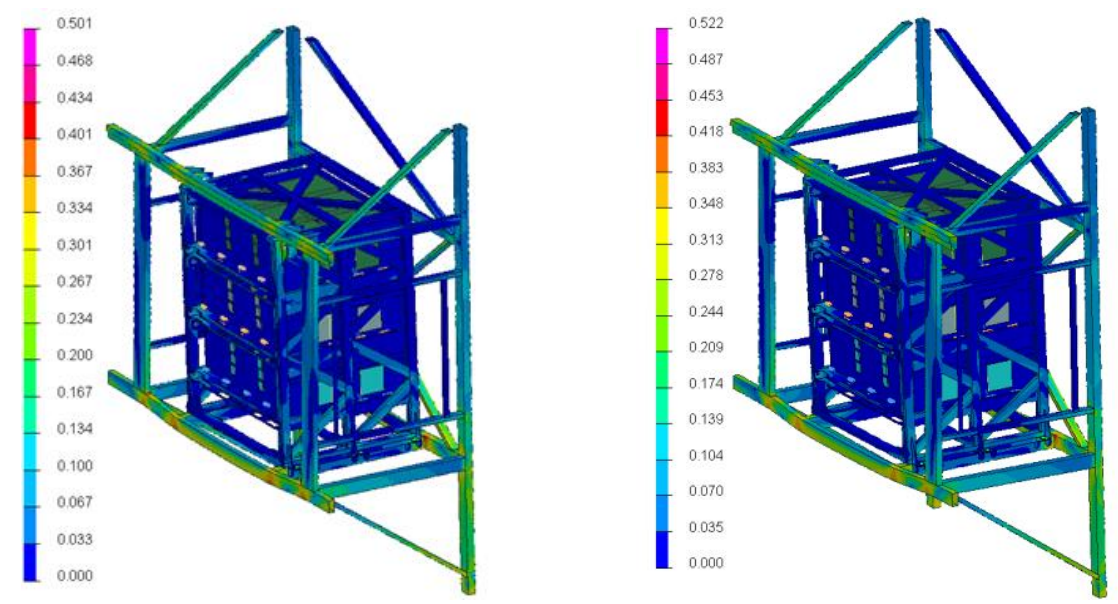

Fig. 4. (a) Von Mises stress [GPa] - material without the strain rate response defined; (b) Von Mises stress [GPa] material with strain rate response.

\section{Effects of dynamic forces}

The effects of dynamic forces are significant under loads which vary over a short time. The time of the presently simulated process is $150 \mathrm{~ms}$, which illustrates the significant velocities involved. It can be inferred that inertia may play an important role here. In order to eliminate work hardening at higher velocities, the material model without the strain rate response was used. When static loading is assumed, the rate of the increase in load due to acceleration to the maximum level is very slow. Therefore, a steady-state problem can be formulated. To approximate a quasi-static state, computation with a 
gradual increase in acceleration over $2000 \mathrm{~ms}$ has been created. The node of the REESS which experienced the largest displacement has shifted by $54 \mathrm{~mm}$. This translates into a velocity of approximately $0.027 \mathrm{~m} / \mathrm{s}$ over the time of $2000 \mathrm{~ms}$ (or less, at smaller displacements). During the electric bus crash, the maximum load is reached within 90 ms. The velocity of the node with the largest displacement thus equals $0.6 \mathrm{~m} / \mathrm{s}$. This movement is associated with a substantial amount of energy. Therefore, there is a significant difference between the static and dynamic states. The maximum plastic strain is $0.069 \mathrm{~mm} / \mathrm{mm}$ (Fig. $5 \mathrm{a}$ ), which is in contrast with the maximum plastic strain during the crash of $0.195 \mathrm{~mm} / \mathrm{mm}($ Fig 5 b).
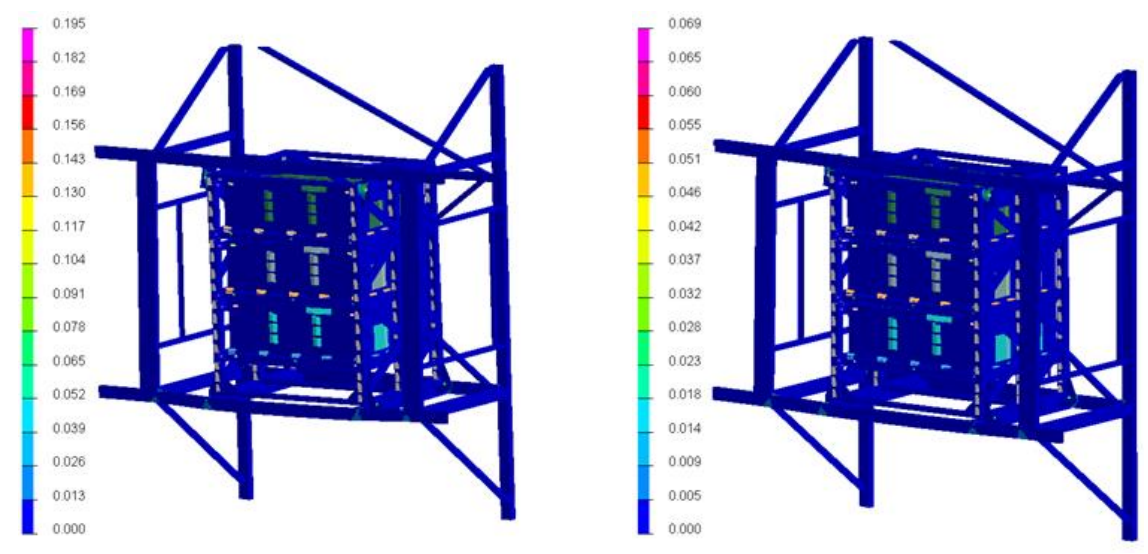

Fig. 5. (a) Maximum plastic strain [-] during crash; (b) Maximum plastic strain [-] under quasi-static load.

\section{Conclusion}

The main point of this paper is to describe the behaviour of the front REESS during battery-electric bus crash using simulations. The simulations show that plastic deformation only occurs locally and that the plastic strain levels are small. This suggests that a road accident of the electric bus will not cause (in the locations investigated) any loss of structural integrity. The calculations offer an opportunity to study various influences. The high-strain rate behaviour is a very interesting aspect. The strain rate in the simulation is approximately $50 \mathrm{~s}-1$. However, the simulations suggest a rather small effect of the strain rate. This is probably the result of relatively small plastic deformation which occurs only locally. Much more significant is the influence of dynamic loading. The initial simulation of a crash yields a much higher maximal plastic strain than the quasi-static loading solution does. It is therefore clear that it is not possible to obtain reliable results using the standard static analysis. The effects of materials plasticity have not been accounted for because it is clear that linear idealization of materials behaviour is not adequate here, due to the occurrence of local plastic deformation.

\section{Acknowledgements}

The present research has been supported under the TA03031347 project sponsored by the Technology Agency of the Czech Republic.

\section{References}

[1] R. Kühne, Electric buses - An energy efficient urban transportation means. (2010) Energy, 35 (12), pp. $4510-4513$.

[2] A. Caragliu, et al., Smart Cities. International Encyclopedia of the Social \& Behavioral Sciences (2015): 113-17.

[3] W. Wen-wei, et al., Pure Electric Bus Crashworthiness Analysis. International Conference on Mechanical Engineering and Material Science (MEMS 2012).

[4] T. Gernot, W. Leitgeb, Battery Modelling for Crash Safety Simulation. Automotive Battery Technology Springer Briefs in Applied Sciences and Technology (2014): 19-35. Web.

[5] T. Belytschko, et al., Explicit algorithms for the nonlinear dynamics of shells. Computer Methods in Applied Mechanics and Engineering 1984; 42:225-251.

[6] P. Verleysena, et al., Effect of strain rate on the forming behaviour of sheet metals, Journal of Materials Processing Technology, 2011

[7] B. Rossi: Mechanical behavior of ferritic grade 3Cr12 stainless steel - Part 2: Yield locus and hardening laws, ScienceDirect 2010

[8] S. Münstermann, et al., Future design methods for optimised use of modern steels in pressure vessels, Dillinger Pressure Vessel Colloquium, September 16th \& 17th, 2009.

[9] Regulation No. 100 Revision 1 - Amendment 2. Uniform provisions concerning the approval of vehicles with regard to specific requirements for the electric power train. UNITED NATIONS, 12 August 2013.

[10] Electrobus Škoda Perun. Škoda. N.p., n.d. Web. 22 Apr. 2015. <http://www.skoda.cz/en/products/electric-andhybrid-buses/skoda-perun/>. 